\title{
Dental Education Required for the Changing Health Care Environment
}

\author{
Margherita Fontana, DDS, PhD; Carlos González-Cabezas, DDS, MSD, PhD; \\ Tracy de Peralta, DDS, PhD; David C. Johnsen, DDS, MS
}

Abstract: To be able to meet the demands for care in 2040, dental graduates will need to address challenges resulting from the rapidly changing health care environment with knowledge and sets of skills to build on current standards and adapt to the future. The purposes of this article are to 1) analyze key challenges likely to evolve considerably between now and 2040 that will impact dental education and practice and 2) propose several sets of skills and educational outcomes necessary to address these challenges. The challenges discussed include changes in prevalence of oral diseases, dental practice patterns, materials and technologies, integrated medical-dental care, role of electronic health records, cultural competence, integrated curricula, interprofessional education, specialty-general balance, and web/cloud-based collaborations. To meet these challenges, the dental graduate will need skills such as core knowledge in basic and clinical dentistry, technical proficiency, critical thinking skills for lifelong learning, ethical and professional values, ability to manage a practice, social responsibility, and ability to function in a collegial intra- and interprofessional setting. Beyond the skills of the individual dentist will be the need for leadership in academia and the practice community. Academic and professional leaders will need to engage key constituencies to develop strategic directions and agendas with all parties pointed toward high standards for individual patients and the public at large. This article was written as part of the project "Advancing Dental Education in the $21^{\text {st }}$ Century."

Dr. Fontana is Professor, University of Michigan School of Dentistry; Dr. González-Cabezas is Richard Christiansen Collegiate Professor of Oral and Craniofacial Global Initiatives and Associate Professor, University of Michigan School of Dentistry; Dr. de Peralta is Clinical Associate Professor, University of Michigan School of Dentistry; and Dr. Johnsen is Dean and Professor, University of Iowa College of Dentistry \& Dental Clinics. Direct correspondence to Dr. Margherita Fontana, University of Michigan School of Dentistry, 1011 N. University, Room 2393, Ann Arbor, MI 48109; 734-647-1225; mfontan@umich.edu.

Keywords: dental education, curriculum, skills, education outcomes

Submitted for publication 1/17/17; accepted 2/28/17

doi: 10.21815/JDE.017.022

$\mathrm{T}$ The purposes of this article are to 1) analyze key challenges likely to evolve considerably between now and 2040 that will impact dental education and practice and 2) propose several sets of skills and educational outcomes necessary to address these challenges. This article was written as part of the project "Advancing Dental Education in the $21^{\text {st }}$ Century."

\section{Challenges That Will Drive Change}

The health care environment in 2040 will be considerably different from the one we have today. Targeted, evidence-based, team-based health care delivery is becoming paramount in an environment of increasing health care costs, resource constraints, and improved health expectations. This form of delivery will be particularly important with changes in prevalence and disparities in dental caries, periodontal disease, and oral cancer across U.S. population groups, as well as issues associated with changing demographics, access to care, impact of health care finance and quality monitoring on care delivery, and impact of technology and interprofessional practice on dental education and practice models, among other drivers for change. This changing environment will require dentists with broad knowledge and an updated set of skills to address the needs of society. One way to conceptualize the dentists of the future is to envision the many roles they will be called upon to play, including those of clinician, scholar, manager and/or team leader, communicator, collaborator, and advocate. ${ }^{1}$ We begin by considering examples of challenges and the associated changes 
in knowledge and skills needed in 2040 to address these challenges.

Changes in prevalence of oral diseases. In the last decades, dental caries and periodontal disease trends have shown a slow but steady decrease in prevalence and severity, which is reflected in a significant decrease in edentulism. ${ }^{2}$ Based on these trends, it is reasonable to expect that the general U.S. population in 2040 will have lower levels of uncontrolled oral diseases, pain, and reduced masticatory function. Therefore, the percentage of the population that will need significant regular periodontal and restorative care will be smaller. However, despite these favorable trends, high-risk groups for periodontal disease and dental caries will continue to exist, as health disparities are likely to continue to widen. ${ }^{3}$ Other oral health diseases, such as head and neck cancer, are also present in higher percentages in some groups of the population, frequently with poorer outcomes when treated. ${ }^{4}$ Furthermore, the majority of high-risk individuals have serious limitations in accessing care, a problem not likely to change in the future if we maintain the current care delivery model. Population and disease changes will impact the type of patients available to our educational programs.

Changes in dental practice patterns. Currently in the U.S., more than $\$ 110$ billion is spent in oral care, mostly in private practice settings operating in relative isolation from other health care providers. ${ }^{5}$ This model will not be sustainable in the long run because of cost and because it will not be able to address the oral and overall health needs of the population. Over the last few decades, there have already been noticeable changes in dental practice patterns. Dental service organizations that manage large numbers of dental practices are increasing in size and number, and it is possible to conceive that the days of the solo dental practice are numbered. This is in part a consequence of the cost-efficiencies achievable through large-scale implementation of electronic records and other technologies and of purchasing arrangements. In addition, high levels of student debt contribute to the desirability of these kinds of arrangements, which offer alternatives to the large investment needed for opening or buying into smaller practices. At the same time, new models are evolving that consider the role of dental services in the larger health care system (e.g., dentists integrated into hospitals, teledentistry). It is clear that the dentist of the future must be capable of functioning in a much broader and more complex environment than in the past, and dental education must prepare graduates with the practice management, communication, and leadership skills needed to function in this changing environment. ${ }^{1}$

Changes in communication, management, and leadership skills. Chronic oral conditions such as dental caries and periodontal disease are likely to be managed differently in the future, using a combination of classic and novel nonsurgical and surgical technologies and products. It is not affordable and unnecessary for dentists to provide all phases of care for these chronic dental conditions. The necessary components of chronic care management can be carried out by a team of well-trained health care providers, either within dentistry or across other professions, likely resulting in more cost-effective disease management with better health outcomes. In fact, dental professionals will need to embrace greater leadership roles in the evolving health care environment to advance the oral health of the public. ${ }^{1,6}$ Dentists will need to learn to effectively communicate, manage, and lead intraprofessional dental teams and must also learn to either manage or be an effective team collaborator when in interprofessional teams. They will also need to more effectively communicate with patients and communities. Thus, health care team management and the ability to work collaboratively within and across disciplines will be increasingly important skills for dentists to have.

Evolution in materials and technologies. As materials and technologies evolve over time, it is likely that services provided in a general dental setting will adapt to these changes and evolve to facilitate broader access to some procedures currently in the primary purview of specialists (e.g., broader access to advanced care procedures such as implants, regenerative techniques, $\mathrm{CAD} / \mathrm{CAM}$, digital scanning). As disease rates go down, it is likely that individual expectations for esthetics will also be higher than today, not only in quality but in affordability. These needs will demand future dentists to continue to receive training in technical skills associated with changing materials and techniques.

Moves toward integrated medical-dental care. A major change in dental practice will be its significantly deeper integration into the medical system. We have seen increasing understanding across health disciplines that oral health providers are key members of a multidisciplinary health team. Currently, for many dentists, medical integration is primarily reduced to understanding the role that systemic diseases or conditions (e.g., diabetes and high blood pressure) have regarding the health of oral 
tissues, in the delivery of oral care, or by indirectly supporting general health by helping maintain a healthy oral environment and occasionally initiating or participating in patient referrals across disciplines.

However, in the future, it is very likely that dental teams will be expected to be a much more integral part of the medical system, probably involved in part of the overall early diagnosis, risk assessment, and management of a variety of chronic medical conditions, resulting in lower health care costs and improved health outcomes. As with some oral diseases, management of other chronic diseases already considered global leading causes of death and disability will increase in importance and will require better and more cost-effective management systems that depend on teams of health care providers and even groups of non-health professionals, such as family members and teachers. Moreover, patients and their families will become an increasingly relevant and integral part of the health care team, actively participating in treatment decisions and management. ${ }^{7}$ At the same time, other professions will also increase their participation in the diagnosis, prevention, and referral of oral diseases and work together towards ensuring establishment of medical homes, dental homes, or, in some cases, integrated health homes.

More effective use of electronic health records. The new generation of electronic health records will be expected, among other things, to incorporate the ability to be accessible across professions in different health care settings to allow for integrated care, provide evidence-based tools, and be adaptive, patient-focused, and able to integrate new technologies to facilitate high-quality management of patients. Health record systems will probably integrate text mining and biomedical data (textomics) to help provide individualized medical and dental care, and they will be able to measure numerous health and procedure outcomes in real time, influencing diagnosis and management. ${ }^{8}$ At the same time, these innovations will help to improve quality of care by creating standard processes to reduce reliance on memory, enhancing quality, and anticipating potential safety problems by identifying areas in which structural or training changes may be necessary to attain and maintain high levels of care.

Demographic change. Higher levels of cultural competence and health literacy will be another requirement for the dentist of 2040 to adapt to the increased diversity in the population. ${ }^{7}$ These skills will be essential to properly diagnose and establish successful treatment plans, as well as to manage a diverse group of health care personnel. A major shift in the composition of our society is occurring. World Health Organization projections for 2044 show that more than $50 \%$ of the population may no longer be comprised of non-Hispanic whites and that the percentage of foreign-born individuals will continue to increase. ${ }^{9}$ As the World Health Organization noted, these types of population changes stress the need for health professionals "who are globally competent and locally relevant, able to serve their local communities in an effective manner."

Providing access to appropriate care for all groups in society is a constant challenge. Access to care barriers are not restricted to some groups of our social matrix; they are also a perpetual problem in numerous rural areas. In fact, more than half of rural counties in four U.S. states were found to have shortages of dental health professionals more than a decade ago, ${ }^{10}$ and today this percentage is likely to be higher and spread across most U.S. states. Efforts to reach underserved rural areas are taking place, but their span is limited and with little possibility to expand in the current format. Disparities in rural oral health can be attributed to numerous factors including cost, transportation, education, dentist availability, lack of dentists who accept Medicaid and other subsidized insurance, and absence of coordinated referral systems. ${ }^{11}$

\section{Dental Education Outcomes Needed to Address Changes}

A necessary first step in educating and training the ideal practitioner for 2040, and establishing the needed curriculum design, is to delineate the educational outcomes and characteristics of the needed graduate. ${ }^{12,13}$ Few schools start with the outcomes and work back to design courses and tracks. More often schools start with the current set of courses and try to make them better. The question arises: if outcomes have not been articulated first, can learning be meaningfully guided and performance assessed? While we have observed that many schools are making advances in developing a culture of inquiry and looking beyond courses and disciplines, the dental education culture remains strongly attached to traditional courses and disciplines. A key assumption to move forward is that while knowledge and technical skills can be more discipline-specific, all 
other needed outcomes cross disciplines and curricular years, requiring better curriculum integration. ${ }^{14,15}$ For example, critical thinking, ethics, social responsibility, treatment planning, communication and leadership skills, risk assessment, technology, decision making, literature search and critique, evidence-based dentistry, interprofessional education, and problem-based learning (PBL) inherently cross disciplines and curriculum years.

While different kinds of outcomes have been sought in dental education, the following spectrum should be considered: core of knowledge in basic and clinical dentistry, technical proficiency, critical thinking skills, ethical and professional values, ability to manage a practice, social responsibility, communication and leadership skills, and ability to function in a collegial professional setting. ${ }^{12,13}$ Many of these skill sets have not been extensively articulated in a form to guide learning and assess performance, except for knowledge and technical skills, which are then the outcomes that dominate what "counts" for graduation.

To train future dentists with the appropriate knowledge and skills required by the society of 2040 , significant change in our dental education system and educational outcomes must occur, examples of which are discussed in the next paragraphs. The change cannot mean merely adding new knowledge, skills, and competencies to existing ones without integral curriculum revision, as additive changes only will result in dental education likely becoming longer (five or six years). Cost of dental education is already a huge challenge, and creative new solutions are needed if we expect to train dentists for 2040 without significantly increasing costs and student debt. A new financing model, probably with public subsidies, will be needed. On the bright side, new models of health management may demand fewer dentists per unit of population, making this change possible. Another important change in the education of the future dentist is the training environment. Most of the clinical training could be moved from dental schools to facilities such as community clinics, where this more complex and integrated medical-dental care will be occurring.

\section{Integrated Curricula}

Back in 1995, the Institute of Medicine pointed out that dental education was being challenged with fragmented, outdated, redundant content and that part of the problem was that content delivery was coming from isolated disciplinary silos. ${ }^{16}$ Responsive curriculum reform has been made since that report, yet there is much more to be done. One of the proposed changes is the need for horizontal as well as vertical integration models to enhance appreciation of diverse content across disciplines and provide skill-building in conceptualizing content (rather than memorizing content) across disciplines to understand how best to incorporate it during clinical decision making to improve patient care. ${ }^{17}$ Thus, a paradigm shift is needed from the knowledge-technical and course-department orientation now dominant in dental education. By advancing dental curricula through content integration, different learner models have been developed, albeit not commonly used yet. Such models include, for example, immersed models (completely studentcentered for student immersion of content integration) and networked models (integration requiring external networking outside of dentistry), which take the learner beyond the immersion level of learning, preparing students for interprofessional patient care. ${ }^{18}$

In addition, awareness of personalized medicine has expanded as we apply new knowledge and skills in risk assessment to determine appropriate individualized, patient-centered preventive and/or interceptive dental care. ${ }^{19}$ This push towards implementation of targeted interprofessional care (IPC) is creating an expanding need among dental and medical educators and practitioners for the development of evidence-based clinical guidelines and necessary infrastructure for implementation. Dental schools will have an increasingly critical role to play in clinical guidelines development and implementation.

\section{Interprofessional Education}

Interprofessional education (IPE), as well as increased intraprofessional education among students in the various areas of oral health, has become a priority in dental curriculum reform, ${ }^{20}$ which will continue and expand in the future. The growing body of evidence on the connections between oral and general health will drive collaborations among health professionals to improve health, with an emphasis on better defining educational outcomes associated with grasping the roles and perspectives of every member of the health care team in making better integrated decisions for patient and community care. IPE can be delivered and outcomes measured in multidisciplinary didactic courses, observations in various practice settings, and IPC models. While we already have competencies for dental education 
associated with IPE, ${ }^{21}$ explicit outcomes (skill sets) have not been articulated for the practitioner. Assessment strategies are still in the early stages to be included in planning and executing care in medicine, pharmacy, nursing, dentistry, physical therapy, nutrition, and social work.

Interprofessional and integrated practice and education models will require considerable changes in the clinical practice environments, changes in reimbursement models (some already facilitated by current health care reform), definition of relevant and measurable oral health outcomes, and clinician skill-building in intra- and interprofessional communication and collaboration. It is possible that many health care programs could decrease costs by minimizing duplication of efforts, but a major obstacle to getting started with curricular redesign is identifying faculty members with the appropriate training and experience in health system science to implement the changes. ${ }^{7}$ Thus, programs for faculty training and calibration need to start now and be adaptable to future needs, and faculty members may need to function across disciplines and maybe even across dental schools.

\section{Critical Thinking and Problem- Solving}

Critical thinking and problem-solving are likely to continue being two of the most desirable goals and needed measurable outcomes of student learning across disciplines and professions. Critical thinking involves scrutinizing, differentiating, and appraising information/evidence, as well as reflecting on information to make judgments that will help problem-solve and inform clinical decisions. In fact, in order to adequately assess outcomes in critical thinking, dental education will need to expand current competencies in reflective practice to include reflections associated with IPC. ${ }^{22}$ Furthermore, basic concepts in critical thinking are the first part of the progression to demonstrating patient-relevant critical thinking skill sets. An emerging set of concepts from the education literature centers around emulating the intended activity, including 1) engaging the student in multiple situations calling for structured critical thinking; 2) emulating the intended activity for validity, ${ }^{23,24}$ which increases with directness, transparency, and utility; 3) gaining agreement of experts (faculty) on the content, delivery, and assessment for reliability; ${ }^{23} 4$ ) using the same instrument to guide learning and assess performance throughout the curriculum; and 5) including alternatives, biases, and self-assessment. ${ }^{25}$ While critical thinking skills have been defined, no unifying skill set has yet been developed. Examples of critical thinking skill sets have been reported for treatment planning, search and critique of literature, evidence-based dentistry, reflections, caries risk assessment, behavior management, geriatric risk assessment, and technology decision making. ${ }^{26-30}$

A focus on learning to critically think and problem-solve in any discipline, especially across disciplines and professions, requires a different way of teaching and assessment. New models are currently being reported to address the necessary improvement of conceptual learning for applying critical thinking to clinical decision making vs. content memorization. ${ }^{31}$ If the goal is for students to use critical thinking skills, then opportunities must be provided to engage them in PBL, analyze case-based scenarios, and involve them in debates, role-plays, argument mapping, thinking aloud, and simulation, among other activities, while also modifying competency assessment strategies. ${ }^{2}$ Such learning and assessment models should be developed around student-led approaches that support adult learning. ${ }^{32}$

The educational setting also contributes to a culture for critical thinking. A trend to small groups in PBL, ${ }^{33}$ for example, gives students the opportunity to engage one another and faculty members in critical thinking. In some cases, student-led approaches have been expanded to student-led clinics for patient care, thus challenging students to critically think from both a patient care perspective and a financial model perspective. ${ }^{34}$ Some groups are approaching this challenge by creating workflows in student clinics that require a combined knowledge of clinical risk assessment across disciplines and individual patient need measurements in clinical settings. ${ }^{29}$ This reinforcement of critical thinking while in clinical care will better prepare students to work in sustainable intra- and interprofessional models of patient care.

In addition, essential to developing critical thinking skills is a culture with a substantive, engaged faculty in a respectful environment. ${ }^{21,35}$ Observing (and sharing) the thought process with the student offers directness for validity, but the pedagogy of delivery for explicit skill sets associated with critical thinking is in its infancy. Assessment is formative with a degree of precision adequate to determine a level of performance deemed as competent but not precise enough to rank students. Other useful tools reflecting critical thinking are more indirect, for 
example, objective structured clinical examinations (OSCEs), algorithms, and multiple-choice tests. ${ }^{36}$ Since there are many alternatives more often than one "right" answer in clinical care, critical thinking in the health sciences focuses on alternatives, biases, and self-assessment. ${ }^{24}$ The thought process (what students are to do and how they reach and justify an appropriate answer) becomes the outcome. Critical thinking does not fit neatly into courses and departments, thus challenging a culture built around courses and departments, knowledge, and technical skills. ${ }^{14} \mathrm{An}$ inherent challenge in assessment of critical thinking is that there is no body of scholarship for the accreditation standards, so there is no peer-reviewed prototype and no reference point that can be used in education or to calibrate accreditation site visitors..$^{20,37}$

\section{Need for Lifelong Learning}

The integration of conceptual understanding and skill-building in reflective practice, critical thinking, evidence-based dentistry, and clinical leadership has the ultimate goal of producing lifelong learners, as individuals progress through their dental education and practice journey. With the complex and voluminous evidence published regularly for the benefit of patient care today, skills in lifelong learning are among the most essential skills students can gain to remain competent health care providers after their formal education is complete. Instilling an understanding of and perceived value for lifelong learning can be challenging. Complexities in student attitudes toward lifelong learning that have been recognized encourage development of approaches to create student buy-in for need of lifelong learning in their future professional lives. ${ }^{38,39}$ Student-led approaches are a vital component to adult learning and will require reformed curriculum content and systems. ${ }^{40}$

\section{Continued Importance of Ethical and Professional Values}

Ethics is an inherent part of being a professional and the pillar for public trust. Knowledge of ethical principles and the ability to apply ethical concepts to individual patient cases are important outcomes that need to be assessed in dental education. ${ }^{41}$ Ethics is a subset of critical thinking that includes biases and reflection. A more general outcome is the trust of the public, which starts with a wide perception that dentistry is an ethical profession. Knowledge in principles of ethics is widely accomplished in dental education. Assessment can be achieved using objective tests for knowledge demonstration as well as skill set demonstration by application to individual patients. However, there is no widely accepted practical skill set to demonstrate for every patient. Thus, ethics is difficult to assess in daily clinics and is counted less than technical procedures in determination of competence. ${ }^{13}$

\section{Focus on Clinical Leadership and Practice Management}

With the awareness of complex patient needs that require team-based approaches to treatment, a current focus on clinical leadership in dental education can be observed globally across clinical curricula. Competence in clinical leadership involves both conceptual understanding and skill development in relationship-building, facilitation, negotiation, communication, and development of emotional intelligence. ${ }^{42}$ However, challenges in best practices for learning and assessment programs remain and will need to be further developed in the future.

Furthermore, the scope of dental practice will continue to evolve. New dollars in dentistry are provided for children, elderly, and Medicaid expansion, with a drop for employed adults. For example, dental therapists may become more prevalent once dentistry resolves the perceived philosophical aspects and realizes a diversity of team members may contribute to a busy practice that is financially successful. An important educational outcome will be associated with knowledge of factors affecting practice management and experience in functioning in diverse practice settings. Team function, communication, practice settings, payment models (fee for service, managed care), scope of practice, and IT/digital resources are all part of effective practice management. Assessment of these outcomes should be based on demonstration of knowledge on objective tests and participation in care delivery in contemporary settings.

\section{Social Responsibility and Ability to Function in Collegial Settings}

Awareness of societal needs and current policy for oral health, as well as experience in providing care to underserved populations, is necessary. With dramatic changes continuing in the demographics of society, cultural competence is essential. Dealing with one's biases is an inherent part of inclusion and dovetails with the systematic consideration of one's 
biases as an integral part of critical thinking. ${ }^{25}$ Thus, an important outcome for dental education is and will continue to be the need for knowledge on matters of access and disparities, as well as opportunities to participate individually in policy matters. Assessment needs to involve knowledge demonstration on objective tests, participation in delivering care to the underserved, and involvement in organizations and agencies affecting policy. An increasing challenge is that the number of diverse underserved populations is large, while curriculum time is limited. Therefore, outcomes are still elusive, as is making this area "count" toward competence.

\section{Core Knowledge and Discipline- Specific Technical Skills}

Basic and clinical knowledge and technical skills (i.e., demonstrated skills in restorative dentistry, endodontics, oral surgery, periodontics, and other areas) are deeply rooted essential outcomes in dental education, greatly influencing the way information is transmitted (generally to large groups) and the way performance is assessed (generally with objective tests). ${ }^{43,44}$ Technical skills are generally assessed following criterion-referenced clinical measurement followed by evaluation of grasp of concepts. Knowledge can be gained in lectures or electronically (e.g., websites, MOOCs) and is one area where efficiencies in education can be achieved, whereas technical skills are delivered first in the laboratory and ultimately in the clinical setting. Because of the relative precision in assessment, knowledge and technical skills "count" greatly in determining competence and ranking students. Technical skills (i.e., procedural knowledge) and knowledge are subsets of the same outcome, and a dilemma is that a single course can decide which procedures are taught and how they are assessed, thus contributing to fragmented knowledge. Overall technical competence could be determined additively, as with knowledge, with little coordination except to minimize redundance. With the explosion of new technology, decision making will be key (see section on critical thinking).

\section{Changes in Materials, Technologies, and Scope of Practice}

In the last century, there have been many advances in dental materials and associated procedures and equipment/technologies, and these changes are expected to continue in the future, dramatically ex- panding the number of options available for preventing and restoring the consequences of dental diseases. For example, today we have increased understanding of the importance of oral health care to preserve tooth structure and to be as minimally invasive as possible. We have better composite materials being used routinely also for posterior restorations in high stress areas. We have an explosion of new ceramic materials and fabrication technologies hoping to become the mainstays for future restorative dentistry. All of these compete with readily available implant systems and tissue-engineering opportunities. ${ }^{45}$ The Minamata Convention has agreed to a worldwide reduction and ultimate elimination of the production and use of mercury-containing products, which will have significant implications for dental practice by eventually eliminating or making significantly more difficult the use of dental amalgam. ${ }^{46}$ Furthermore, resin composite has replaced amalgam as the material of choice (both by providers and patients) for most clinical situations.

These changes in practice preferences, material availability, and research outcomes for newer materials and techniques need to be reflected in dental education, both in terms of curriculum time and competency assessment. In addition, as trends in oral diseases change in the U.S. and financial constraints magnify issues associated with educational debt for dental professionals, the practice of dentistry is changing, and dental procedures that were once the domain primarily of specialties are penetrating into general practice in increased numbers (e.g., implants).

\section{Web/Cloud-Based Collaborations for Education and Practice}

Educators have only a certain amount of time to deliver a curriculum that provides students with the knowledge and experience they need to become competent practitioners, and the focus should be on finding the most effective pedagogy that maximizes opportunities and available technology. ${ }^{7}$ Technology plays a key role in the development of strategies to improve dental education and practice. Electronic textbook programs, e-grading, digital imaging and impression taking, surgical laser technologies, 3-D printing, and virtual dental simulation are examples of advanced technologies in recent decades that have been introduced in dental education. ${ }^{47-49}$

Interestingly, we have observed that one of dental students' main concerns today in choosing a dental school for their education is if the school will support 
their learning advanced uses of technology for ideal patient care. The Internet is also quickly changing the face of content delivery and assessment strategies for clinical education. This acceleration of change is sure to continue in the next decades, and grasping its potential as an educational resource is vitally important to our future dental learners. The use of such systems as educational forums, electronic health record keeping, dashboards of clinical competency progression, skill-building portfolio development, student-produced skill progress-reporting websites, evidence-based dental education portals, and many other applications to come will advance our future educational and clinical practices in new forms that we cannot imagine today. ${ }^{13,50}$ Finally, as we consider the characteristics of current generations, who will be the majority of the faculty in 2040, and extrapolate to the future students of 2040, consideration will need to be given to preferred modes of communication, which should then inform curriculum strategies on how to best engage participants in learning and in continuing education efforts.

\section{Financing of Dental Care}

While it is beyond the scope of this article to offer an analysis of the financing of dental care, some basic assumptions will frame the issues and skills needed to adapt. One assumption is that a large part of dental care will be financed by self-payment from individual patients. This practice is fundamentally different from medicine, in which a much smaller part of medical care is financed out-of-pocket, both in hospitals and in physicians' offices. Dental education and practice will need to adapt to this changing financial model of dental care, and practice management skills will continue to be an important need for dental graduates.

\section{Conclusion}

This article discussed examples of knowledge and skills that dentists will need to have to effectively and efficiently function in the changing health care environment and meet the demands for care in 2040. It also proposed some changes needed in dental education to meet those future needs.

\section{Editor's Disclosure}

This article is published in an online-only supplement to the Journal of Dental Education as part of a special project that was conducted independently of the American Dental Education Association (ADEA). Manuscripts for this supplement were reviewed by the project's directors and the coordinators of the project's sections and were assessed for general content and formatting by the editorial staff. Any opinions expressed are those of the authors and do not necessarily represent the Journal of Dental Education or ADEA.

\section{REFERENCES}

1. Berg JH, Mouradian WE. Integration of dentistry and medicine and the dentist of the future: changes in dental education. J Calif Dent Assoc 2014;42(10):697-700.

2. Dye BA, Tan S, Smith V, et al. Trends in oral health status: United States, 1988-94 and 1999-2004. National Center for Health Statistics. Vital Health Stat 2007;11(248).

3. Capurro DA, Iafolla T, Kongman A, et al. Trends in income-related inequality in untreated caries among children in the United States: findings from NHANES I, NHANES III, and NHANES 1999-2004. Community Dent Oral Epidemiol 2015;43:500-10.

4. American Cancer Society. Cancer facts and figures. Atlanta: American Cancer Society, 2015.

5. Wall T, Nasseh K, Vujicic M. U.S. dental spending remains flat through 2012. Research Brief, Health Policy Institute, American Dental Association. Chicago: American Dental Association, 2014.

6. Mouradian WE, Lewis CW, Berg JH. Integration of dentistry and medicine and the dentist of the future: the need for a health care team. J Calif Dent Assoc 2014;42(10):687-96.

7. Cuff PA, Global Forum on Innovation in Health Professional Education, Board of Global Health, Institute of Medicine, and National Academies of Science, Engineering, and Medicine. Envisioning the future of health professional education: workshop summary. Washington, DC: National Academies Press, 2015.

8. Reynolds PA, Harper J, Dunne S. Better informed in clinical practice: a brief overview of dental informatics. Br Dent J 2008;204:313-7.

9. World Health Organization. Transforming and scaling up health professionals' education and training: World Health Organization guidelines. Geneva: World Health Organization, 2013.

10. Committee on the Future of Rural Health Care, Board on Healthcare Services. Quality through collaboration: the future of rural health. An Institute of Medicine Report. Washington, DC: National Academies Press, 2004.

11. Fos P, Hutchison L. The state of rural oral health. Rural Healthy People 2010;1:199-203.

12. American Dental Education Association. ADEA competencies for the new general dentist; definition of critical thinking. J Dent Educ 2016;80(7):825-8.

13. Schneider GB, Cunningham-Ford MA, Johnsen DC, et al. Outcomes mapping: a method for dental schools to coordinate learning and assessment based on desired characteristics of a graduate. J Dent Educ 2014;78(9):1268-78. 
14. Johnsen DC, Marshall TA, Finkelstein MW, et al. A model of student learning: a matrix of educational outcomes versus methodologies. J Dent Educ 2011;75(2):160-8.

15. Johnsen DC. Critical thinking: focal point for a culture of inquiry. In: Boyle C. Student learning: improving practice. Hauppauge, NY: Nova Science Publications, 2013: 151-70.

16. Field MJ, ed. Dental education at the crossroads: challenges and change. An Institute of Medicine Report. Washington, DC: National Academy Press, 1995.

17. Howard KM, Stewart T, Woodall W, et al. An integrated curriculum: evolution, evaluation, and future direction. J Dent Educ 2009;73(8):962-71.

18. Fogarty R, Stoehr J. Integrating curricula with multiple intelligences: teams, themes, and threads. $2^{\text {nd }}$ ed. Thousand Oaks, CA: Sage Publications, 2007.

19. Giannobile WV, Braun TM, Caplis AK, et al. Patient stratification for preventive care in dentistry. J Dent Res 2013;92(8):694-701.

20. Commission on Dental Accreditation. Accreditation standards for dental education programs. 2016. At: www. ada.org/ /media/CODA/Files/pde.pdf?la=en. Accessed 26 Jan. 2016.

21. Association of American Medical Colleges. Core competencies for interprofessional collaborative practice. At: www.aamc.org/download/186750/data/core_competencies.pdf. Accessed 26 Jan. 2016.

22. Paul R, Elder L. Critical thinking tools for changing your learning and your life. $2^{\text {nd }}$ ed. Upper Saddle River, NJ: Pearson Prentice Hall, 2006:xvii,54.

23. Lane S, Stone CA. Performance assessment. In: Brennan RL, National Council on Measurement in Education, American Council on Education, eds. Educational measurement. $4^{\text {th }}$ ed. Westport, CT: Praeger, 2006:1-112.

24. Johnsen DC, Lipp MJ, Finkelstein MW, et al. Guiding dental student learning and assessing performance in critical thinking with analysis of emerging strategies. J Dent Educ 2012;76(12):1548-58.

25. Kahneman D. Thinking fast and slow. New York: Farrar, Straus \& Giroux, 2011.

26. Johnsen DC, Finkelstein MW, Marshall TA, et al. A model for critical thinking measurement of dental school performance. J Dent Educ 2009;73(2):177-83.

27. Marshall TA, Finkelstein MW, Qian F. Improved student performance following instructional changes in a problembased learning curriculum. J Dent Educ 2011;75(4): 466-71.

28. Marshall TA, Straub-Morarend CL, Handoo N, et al. Integrating critical thinking and evidence-based dentistry across a four-year dental curriculum: a model for independent learning. J Dent Educ 2014;78(3):359-67.

29. Guzman-Armstrong S, Warren JJ, Cunningham-Ford $\mathrm{MA}$, et al. Concepts in critical thinking applied to caries risk assessment in dental education. J Dent Educ 2014;78(6):914-20.

30. Johnsen DC, Shubot DB, Nash DA. Criterion-referenced self-instructional format for teaching child management skills in the clinic. J Dent Educ 1983;47(2):113-14.

31. Marshall TA, Straub-Morarend CL, Qian F, et al. Perceptions and practices of dental school faculty regarding evidence-based dentistry. J Dent Educ 2013;77(2):146-51.
32. Hoogenes J, Mironova P, Safir O, et al. Student-led learning: a new teaching paradigm for surgical skills. Am J Surg 2015;209(1):107-14.

33. Marshall T, Finkelstein M, Cunningham-Ford M. Problem-based learning: a vehicle to teach critical thinking, reading the scientific literature. MedEdPORTAL. 2010. At: services.aamc.org/30/mededportal/servlet/s/segment/ mededportal/?subid=7931. Accessed 17 Jan. 2017.

34. Bostick G, Hall M, Miciak M. Novel clinical learning from a student-led clinic. Clin Teach 2014;11(7):512-5.

35. Berg R, Call RL, Maguire K, et al. Impact of the University of Colorado's advanced clinical training and service (ACTS) program on dental students' clinical experience and cognitive skills, 1994-2006. J Dent Educ 2010;74(4):423-33.

36. Zartman RR, McWhorter AG, Seale NS, et al. Using OSCE-based evaluation: curricular impact over time. J Dent Educ 2002;66(12):1323-30.

37. Johnsen DC, Williams JN, Baughman PG, et al. New dental accreditation standard on critical thinking: a call for learning models, outcomes, assessments. J Dent Educ 2015;79(10):1138-9.

38. Nash DA. The lifelong learning imperative: ends and means. J Dent Educ 1994;58(10):785-90.

39. Polyzois I, Claffey N, Attstrom R, et al. The role of the curriculum and other factors in determining the mediumto long-term attitude of the practicing dentist towards lifelong learning. Eur J Dent Educ 2010;14:84-91.

40. Hsih KW, Iscoe MS, Lupton JR, et al. The student curriculum review: how we catalyze curricular changes through a student-centered approach. Med Teach 2014;1-5.

41. Sharp HM, Kuthy RA. What do dental students learn in an ethics course? An analysis of student-reported learning outcomes. J Dent Educ 2008;72(12):1450-7.

42. Kalenderian E, Skoulas A, Timothe P, et al. Integrating leadership into a practice management curriculum for dental students. J Dent Educ 2010;74(5):464-71.

43. Albino JEN, Young SK, Neumann LM, et al. Assessing dental students' competence: best practice recommendations in the performance assessment literature in predoctoral dental education. J Dent Educ 2008;72(12):1405-31.

44. Hendricson WD, Rugh JD, Hatch DL, et al. Validation of an instrument to assess evidence-based practice knowledge, attitudes, access, and confidence in the dental environment. J Dent Educ 2011;75(2):131-44.

45. Bayne SC. Beginnings of the dental composite revolution. J Am Dent Assoc 2013;144(8):880-4.

46. FDI World Dental Federation. FDI policy statement on dental amalgam and the Minamata convention on mercury: adopted by the FDI General Assembly, 13 September 2014, New Delhi, India. Int Dent J 2014;64(6):295-6.

47. Curnier F. Teaching dentistry by means of virtual reality: the Geneva project. Int J Comput Dent 2010;13:251-63.

48. Feuerstein P, Adams D. New technologies shape the future of dentistry. Dent Today 2015;34(2):88-92.

49. Morrow JA, Pulido T, Smith PB, et al. Effective use of e-grading in the dental simulation clinic. J Dent Educ 2014;78(6):829-37.

50. Arnett MR, Loewen JM, Romito LM. Use of social media by dental educators. J Dent Educ 2013;77(11):1402-12. 